\title{
Characterization and commissioning of the ATLAS micromegas quadruplet prototype
}

M.Bianco

On behalf of ATLAS Muon Collaboration

Micromegas (Micro Mesh Gaseous Detector) chambers have been chosen for the New Small Wheel (NSW) project, the upgrade of the forward muon spectrometer of the ATLAS experiment both to provide precision tracking and contribute to the trigger.

A quadruplet $(1 \mathrm{~m} \times 0.5 \mathrm{~m})$ has been built at the CERN laboratories, it will serve as prototype for the future ATLAS chambers. This detector is realized using resistive-strip technology and decoupling the amplification mesh from the readout structure. The four readout planes host overall 4096 strips with a pitch of $415 \mu \mathrm{m}$.

A complete detector characterization carried out with cosmic rays, X-Ray source and dedicated test beam is discussed. Characterization is done using analog front-end chip (APV25).

The efforts that lead to the chamber construction and the preparation for the installation in the ATLAS experimental cavern are presented.

Finally, an overview of the readout system developed for this prototype, and integration in to the ATLAS Data Acquisition System is provided.

\section{The Micromegas Small Wheel (MMSW) prototype}
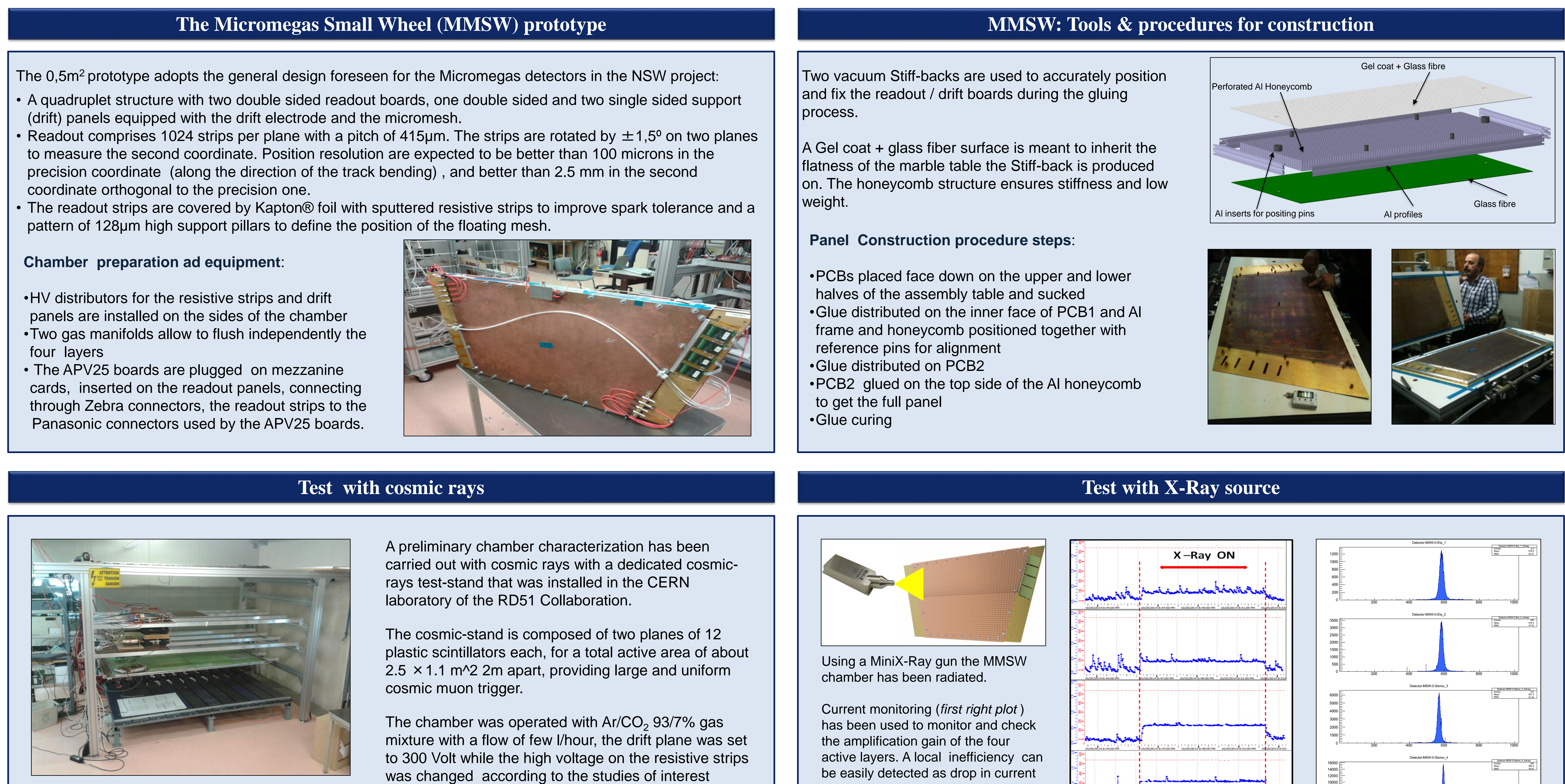

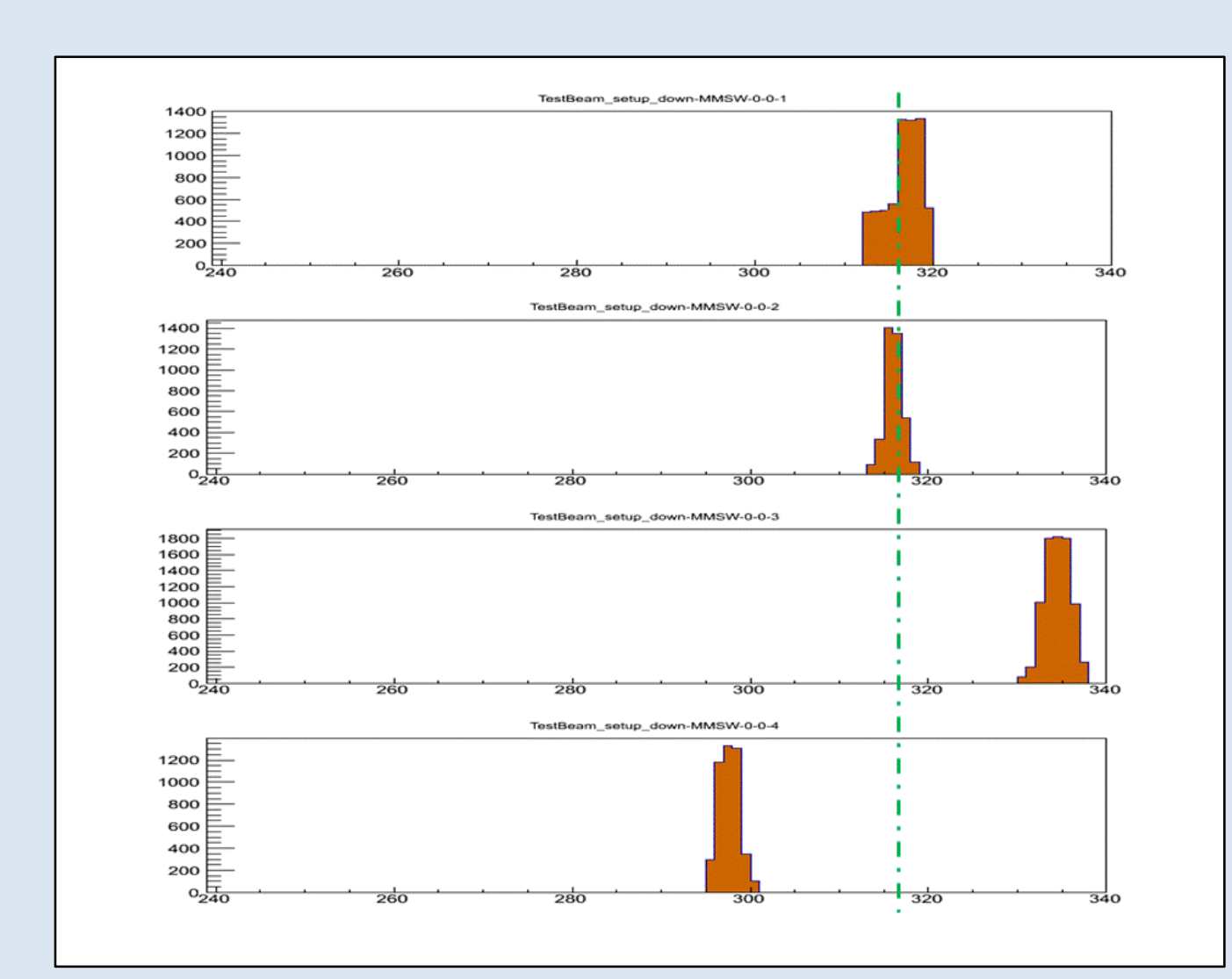

A preliminary chamber characterization has been carried out with cosmic rays with a dedicated cosmicays test-stand that was installed in the CERN laboratory of the RD51 Collaboration.

The cosmic-stand is composed of two planes of 12 plastic scintillators each, for a total active area of about $2.5 \times 1.1 \mathrm{~m}^{\wedge} 22 \mathrm{~m}$ apart
cosmic muon trigger.

The chamber was operated with $\mathrm{Ar} / \mathrm{CO}_{2} 93 / 7 \%$ gas mixture with a flow of few $/ /$ hour, the drift plane was set to 300 Volt while the high voltage on the resistive strips was changed according to the studies of interest Using APV25 front-end ASIC, an SRS based readout system and the dedicated DAQ software MMDAQ3 cosmic rays could be detected in all detector layers.

Full track-events have been observed and successfully reconstructed.

For a vertical track, while the clusters on the first and econd layers look aligned, due to the presence of stereo strips on the third and fourth layers, the

clusters can look to be far from the ideal vertical line. This is an effect of the stereo strips.

The distance of the cluster from such a vertical line is a function of the distance of the incoming particle to the center of the chamber.

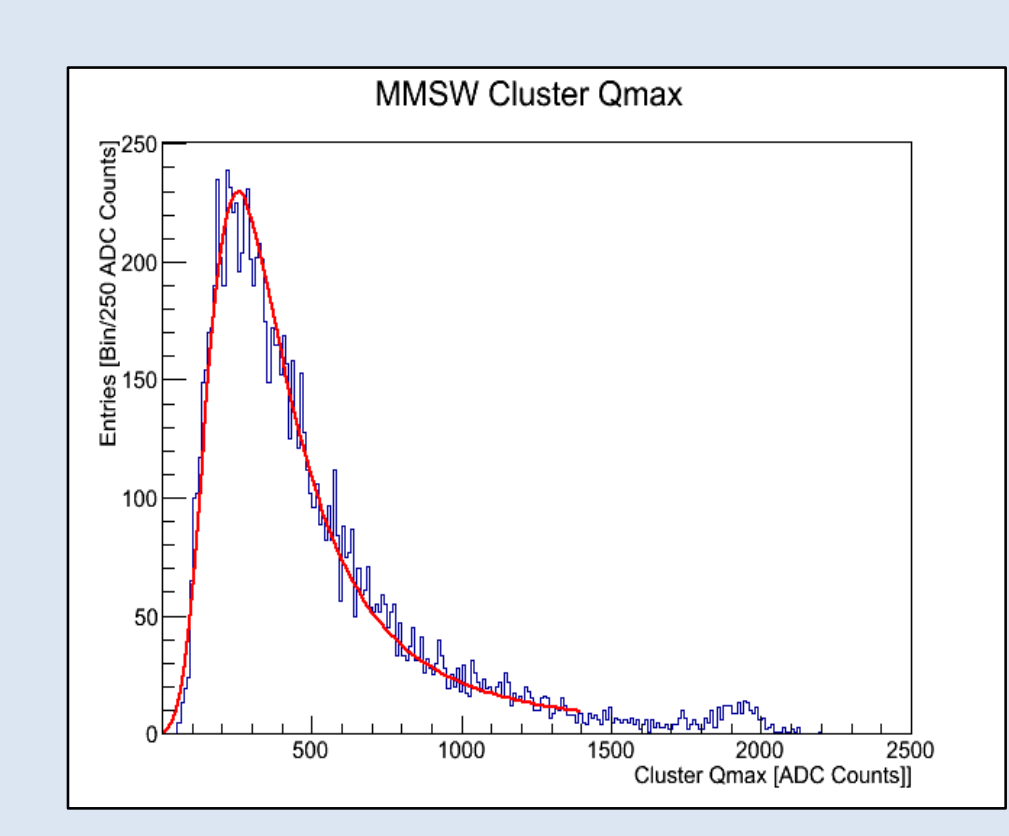

Hits charge distribution, with the typical Hits charge distribution,
APV saturation peak

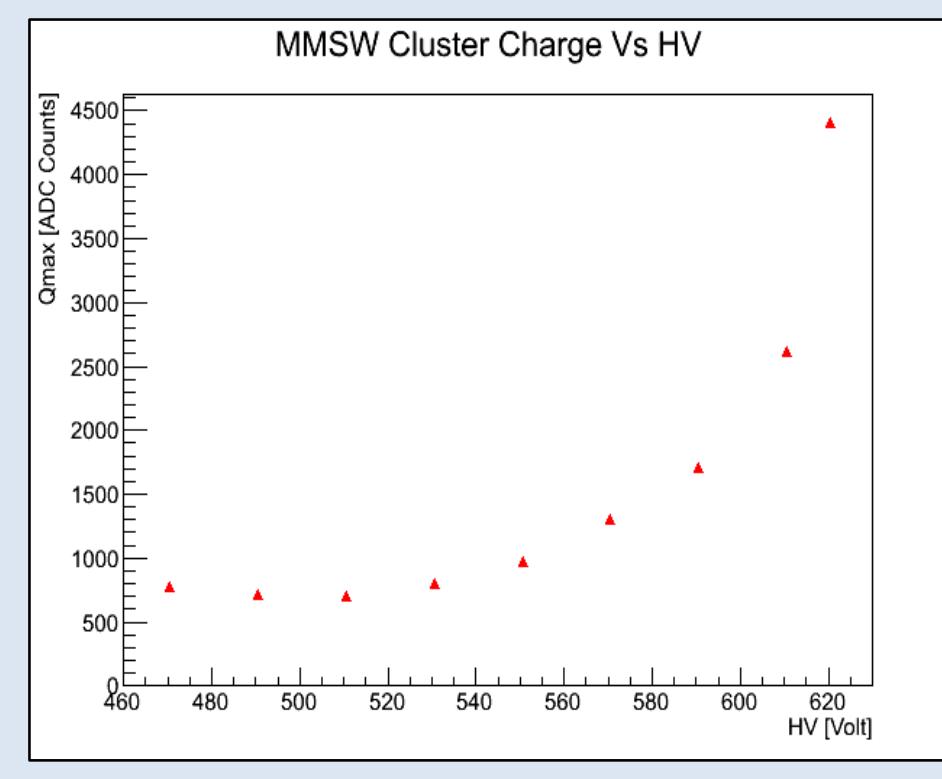

MMSW cluster charge as a function of

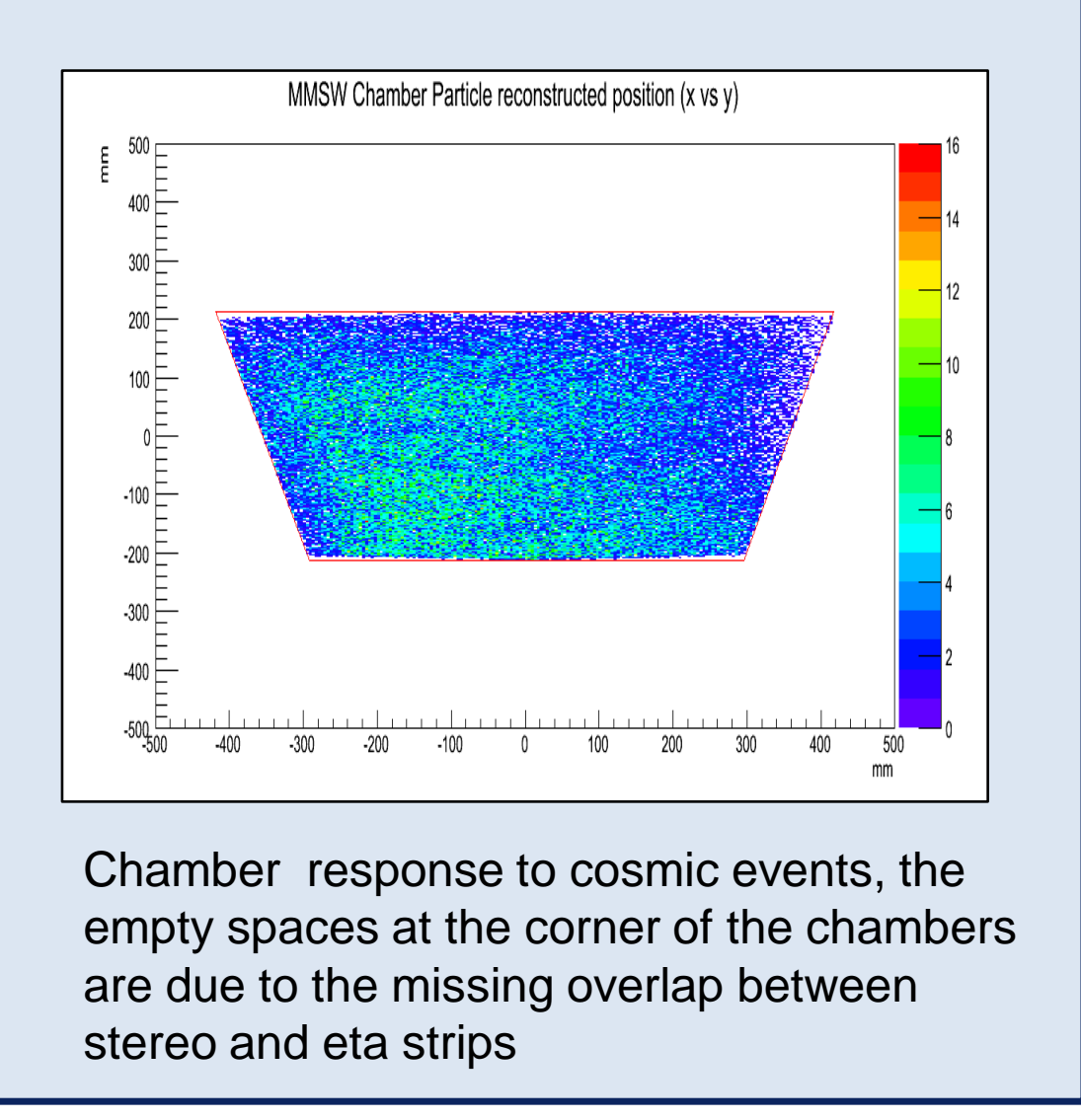

are due to the missing overlap between
sention

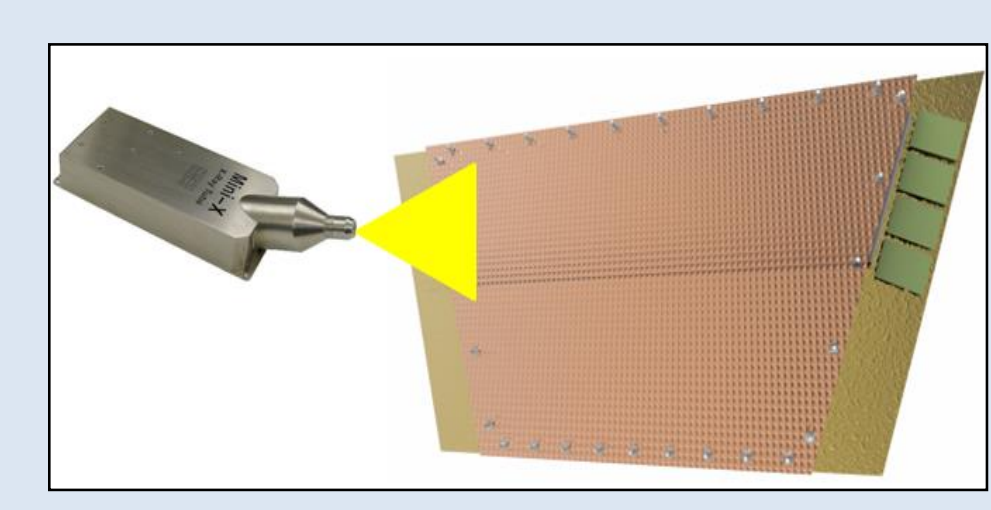

Using a MiniX-Ray gun the MMSW chamber has been radiated.

Current monitoring (first right plot) has been used to monitor and che

active layers. A local inefficioncy be easily detected as drop in current absorption

Collecting data, using front end electronics (second right plot) the relative alignmen
can be checked.

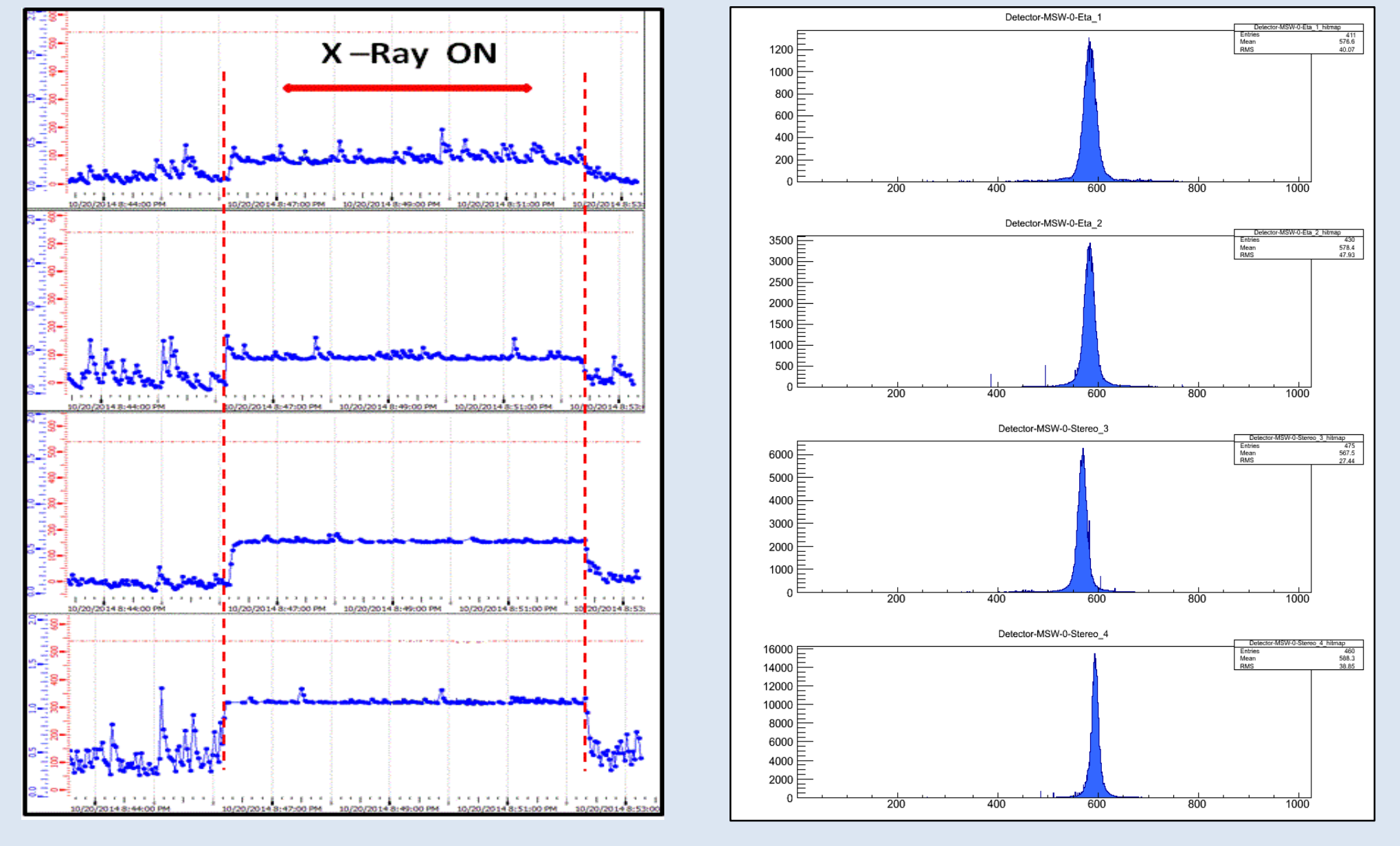

Amplification current, as well as the number of converted photons detected as function of the position of the
plot, farther in the upper plot.

\section{Test beam results}

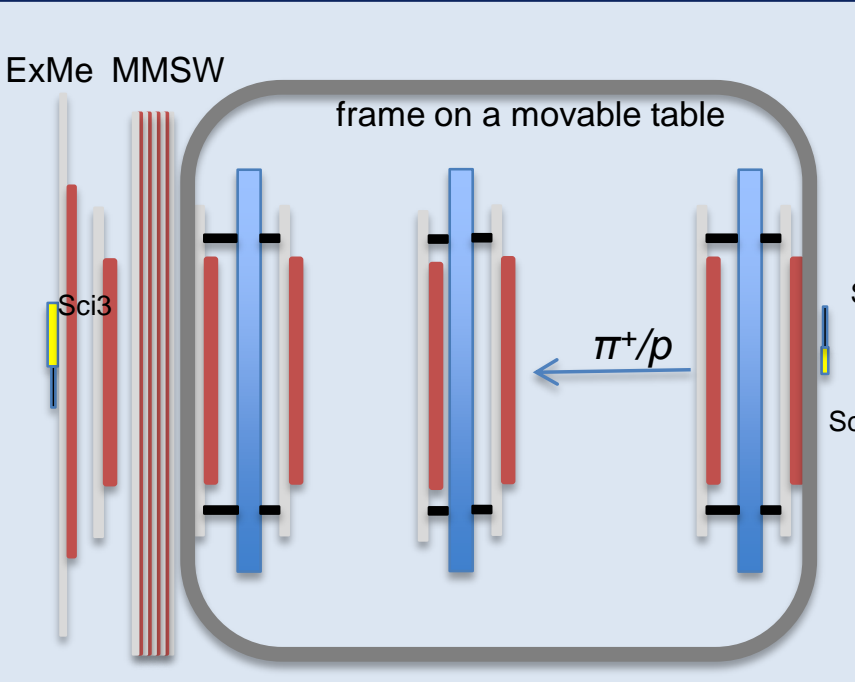

$\mathrm{T}_{\mathrm{Tm} 4} \mathrm{~T} \mathrm{Tmm}$

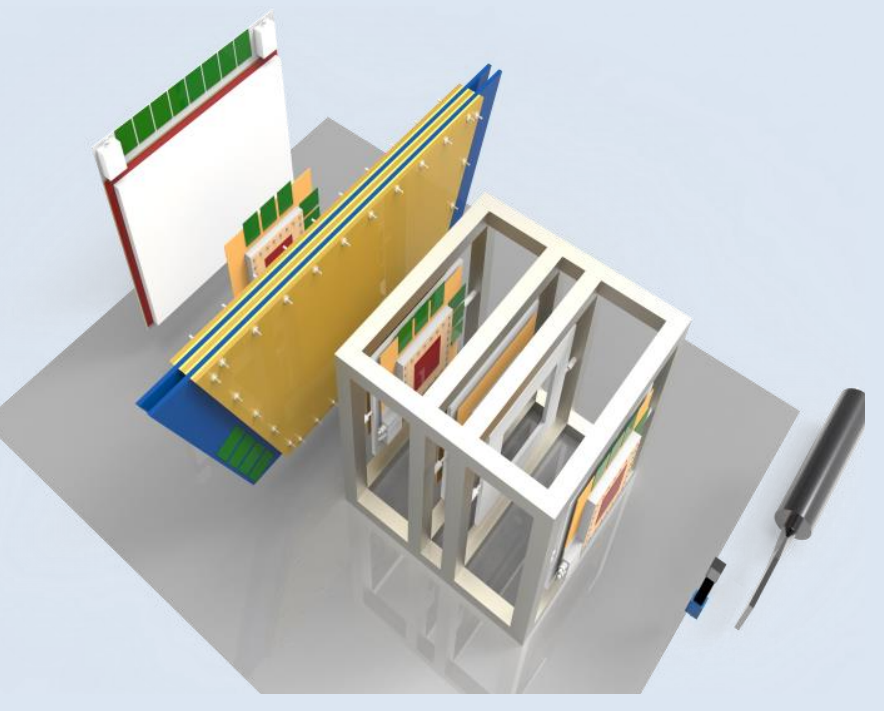

$$
\begin{aligned}
& \text { MMSW chamber has been } \\
& \text { extensively studied during the MMs } \\
& \text { test beam campaign. } \\
& \text { The chamber has been placed } \\
& \text { between an hodoscope, hosting } \\
& \text { several MMs small chambers } \\
& \text { including } 4 \text { double view chambers } \\
& (\mathrm{Tmm}) \text { and a similar chamber } \\
& (\mathrm{Tmm}) \text { placed behind the MMSW } \\
& \text { chamber to reconstruct the incoming } \\
& \text { particles (pions \& protons). } \\
& \text { Due to the } 1.5^{\circ} \text { rotation used to built } \\
& \text { the stereo strip, a resolution on the } \\
& \text { second coordinate is expected to be: } \\
& \sigma_{\mathrm{y}}=27{ }^{*} \sigma_{\mathrm{x}} \text { (right upper plot). } \\
& \text { Resolutions for the precision and } \\
& \text { second coordinate, extracted by the } \\
& \text { test beam data (right lower plots) }
\end{aligned}
$$

\section{The DAQ system for the MMSW into the ATLAS framework}

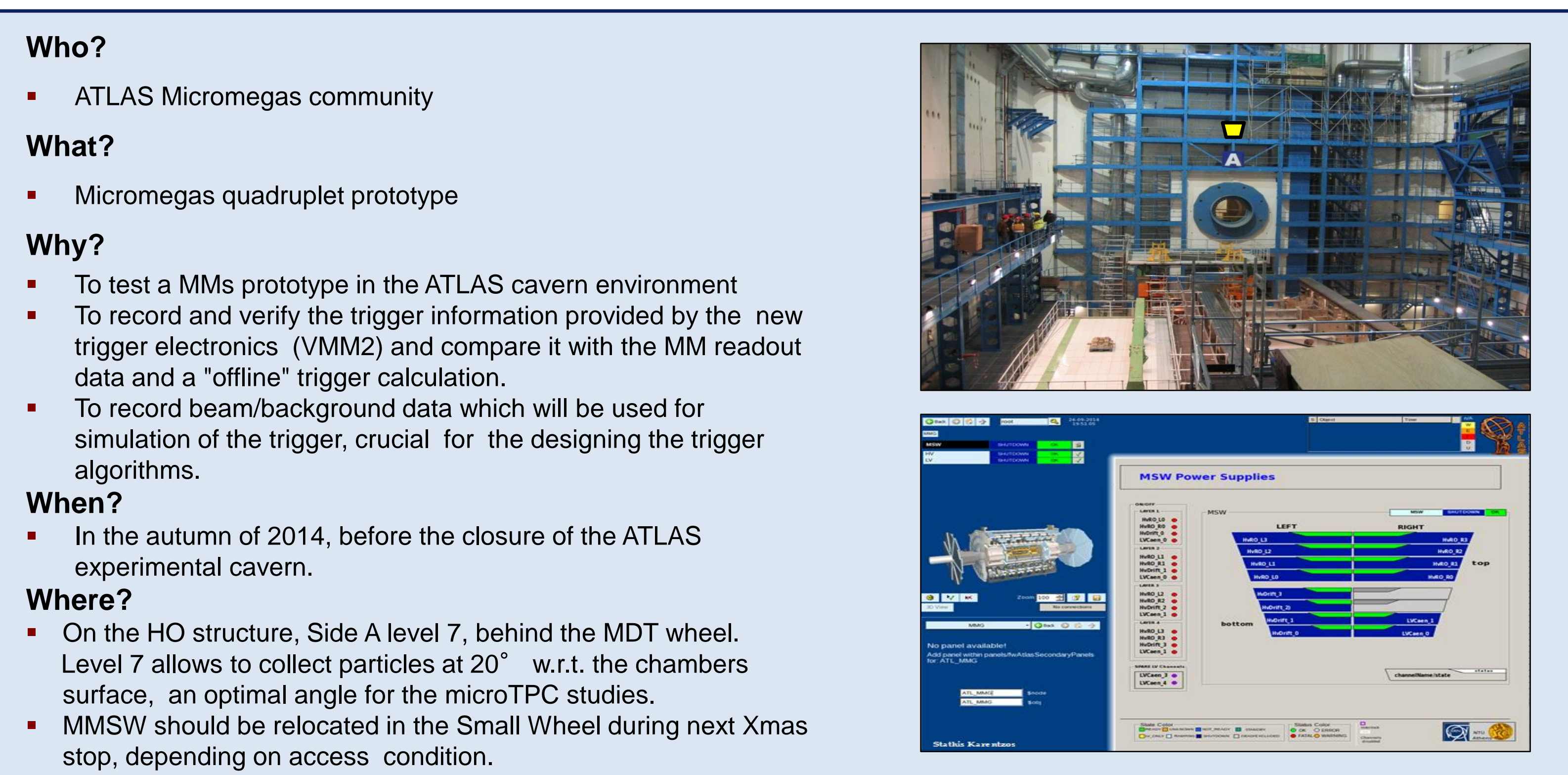

The $4 \times 1024$ strips will be read out by newly developed
VMM2 hybrid chips. Their HDMI output

through optical converter boxes (OC)

Optical fibers will send the data to an ATCA-SRS board in SA15.

A standard Scalable Readout Unit (SRU) will be used in order to get the DTCC link from the ATCA-SRS. The SRU fragments that can be transmitted to the ReadOut System (ROS) via the standard ATLAS readout link (S-Link).

A Micromegas segment has been implemented using the the main ATLAS DAQ partition

MMSW data will be automatically inserted into the ATLAS byte stream

Online software will provide fast detector monitoring

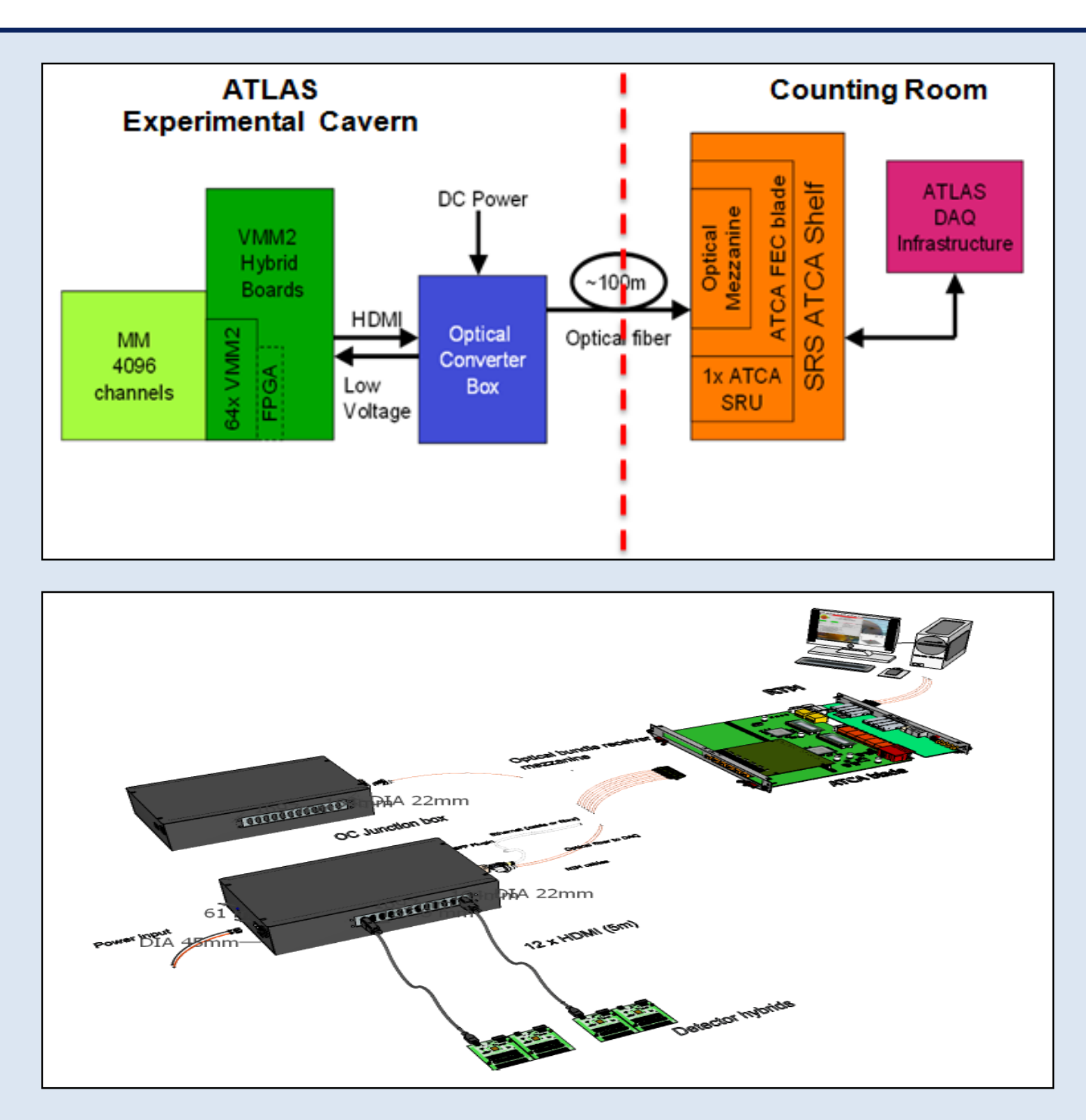

